\title{
Theory-based modeling of LOC-SOC transitions in ASDEX Upgrade
}

\author{
I. Erofeev, E. Fable, C. Angioni, R. M. McDermott and the \\ ASDEX Upgrade Team \\ Max-Planck-Institut für Plasmaphysik, 85748 Garching, Germany \\ E-mail: ivan.erofeev@ipp.mpg.de
}

\begin{abstract}
Modeling of the linear-to-saturated Ohmic confinement transitions is performed on the Ohmic discharges database collected at ASDEX Upgrade. Ion and electron temperatures and densities are predicted using the TGLF turbulence transport model. Self-consistent classical energy equipartition is retained, as well as the impact of low-Z impurities, the core concentration of which is largest at the lowest plasma densities and decreases with increasing electron density. The simulation results are then compared to the experimentally estimated confinement time behavior, and locally to the energy diffusivities behavior across the density ramps. Both density and plasma current are scanned. The key result, which somewhat confirms previous studies, is that the ion energy transport channel is dominated by the ITG instability, which becomes stronger at higher densities due to the reduction in impurity content (as such, reduction in dilution), as well as a moderate increase in ion temperature normalized gradient. A direct impact of the transition between electron modes (TEM) and ITG on the LOCSOC is not observed. However, this is necessary to explain the behavior of the density profile. The linear phase of the confinement is simply attributed to the decrease of electron heat transport as the electron and ion temperatures become more coupled.
\end{abstract}

\section{Introduction}

As has been observed in many tokamaks, the energy confinement of Ohmically heated Lmode plasmas scales linearly with the plasma density [1] until a critical value is reached, after which the confinement stays constants or even degrades, see [2] and references therein. The two regimes are called Linear and Saturated Ohmic Confinement (LOC and SOC). It has been suggested to link them to a shift in turbulence regime from TEM-dominated to ITG-dominated, that modifies transport [3-5]. The critical density of the LOC-SOC transition was found to be close to the value at which the spontaneous plasma toroidal rotation flips from co- to counter-current, and the rollover in density peaking occurs. While it is obvious that the single governing engineering parameter is the plasma density, there is still no definite proof as to whether the three are connected to the same fundamental mechanism, or there are multiple mechanisms at play.

The most important ingredient for the modeling is the correct treatment of turbulence throughout the whole confined plasma, since the dominant mode transition 
from TEM to ITG is believed to begin at the edge and move towards the core (due to higher collisionality and $R / L_{T_{i}}$ at the edge). Along with self-consistent evolution of plasma profiles, such as temperature and density, this poses a demanding simulation task. While modern gyrokinetic codes allow such simulations [6] only at enormously high computational costs, a faster and easier yet sufficiently comprehensive way is implemented in this work. Here, the ASTRA transport code is employed $[7,8]$, coupled to the TGLF transport model [9].

In this work we usually compare three sets of data. One comprises the experimental measurements taken from the AUG Ohmic L-mode database [10]. It is referred to as "Data". Another one consists of the results of ASTRA simulations with experimental kinetic profiles and plasma boundary, and with heat transport coefficients obtained from power balance consideration. This one is referred to as "PB" that stands for power balance. The results of ASTRA simulations with TGLF module and self-consistent evolution of the kinetic profiles, yet with experimental plasma boundary (separatrix coordinates) forms the third data set, which is labeled "TGLF". Both types of ASTRA simulations feature free evolution of current density distribution, and most of the model parameters are the same.

The model is described in section 2. The results of the Ohmic confinement regimes simulation are presented in section 3. Section 4 reports on a dedicated analysis of the turbulence spectra and sensitivity with respect to parameters variation. We demonstrate the effect of impurity content increase on the confinement quality in section 5. General conclusions and prospects constitute the last section 6 .

\section{Modeling assumptions}

\subsection{Model description}

We perform the modeling of a fully ionized plasma with deuterium as main species in ASDEX Upgrade geometry, with Ohmic plasma heating. The experimental basis of the modeling consists of two subsets comprised of two discharges each, for a total of four discharges. The subsets are distinguished by different magnetic fields and plasma currents, while the paired discharges provide a more complete density variation than possible within a single plasma shot. The first subset contains shots \#27000 and \#27001 with $B_{\mathrm{t}}=2.51 \mathrm{~T}$ and $I_{\mathrm{p}}=1.04 \mathrm{MA}$, it features also minor pulsed ECRH with averaged $P_{\mathrm{ECRH}} \approx 130 \mathrm{~kW}$. The second subset is represented by shots \#28386 and \#28243 with $B_{\mathrm{t}}=1.91 \mathrm{~T}$ and $I_{\mathrm{p}}=0.63 \mathrm{MA}$. The aim of such a choice is to check the scaling of transition-relevant quantities with $I_{p}$, as well as to have a two-dimensional basis for parametric sensitivity analysis of heat fluxes (see the last section). The experimental temperature and density profiles are averaged over $\approx 40 \mathrm{~ms}$, when plasma is effectively at equilibrium.

ASTRA solves transport equations for the electron and ion heat diffusion, electron particle diffusion and pinch. The self-consistent evolution of energy transport 
coefficients and plasma temperature profiles saturates when the heating is balanced by outward radial heat fluxes. Turbulent transport coefficients (heat conductivities $\chi_{\mathrm{e}}$ and $\chi_{\mathrm{i}}$, particle diffusivity $D_{\mathrm{n}}$ and convection coefficient $C_{\mathrm{n}}$ ) are computed by TGLF every fifth time step on the basis of evolving kinetic profiles in ASTRA, and are then plugged back, resulting in full self-consistency. Neoclassical transport coefficients are computed with analytical expressions [11]. The two sets of transport coefficients then sum up linearly.

The electron diffusion and convection coefficients are balanced by a particle source that consists of a gas puffing and an ionization model. The puffing rate adjusts itself, so that the line integrated electron density stays equal to the experimental value. Boron is used as the only impurity species as it is intrinsic to AUG plasmas, where the wall undergoes frequent boronizations. Mean boron concentration follows the scaling $Z_{\text {eff }}=1+3.2 I_{\mathrm{p}}^{3} / \bar{n}_{\mathrm{e}}^{3}$ with $I_{p}$ expressed in $M A$ and $\bar{n}_{\mathrm{e}}$ in $10^{19} \mathrm{~m}^{-3}$, and is further discussed in section 2.2. The density of main plasma ions and boron impurity ions is computed from the quasineutrality condition and $Z_{\text {eff }}$ according to the scaling.

The boundary condition for the electron density is set at the separatrix by an outflow proportional to the local density value. Boundary conditions for the temperatures are set at the separatrix, as well. Since the error bars on the edge temperature measurements are of the order of the measured values, we used a scaling that approximates the experimental values, see fig 1 . It consists in inverse proportionality of the edge electron temperature to the edge electron density that has been shown valid [17], and $T_{\mathrm{i}, \rho=1}=2 T_{\mathrm{e}, \rho=1}$. The plasma boundary shape is defined by
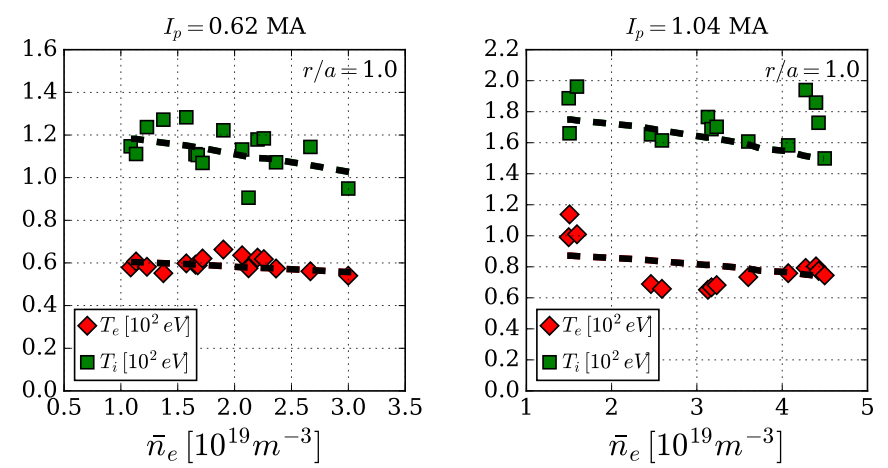

Figure 1. Electron and ion temperature boundary conditions at the plasma edge. The dashed curves represent the scaling used in the modeling.

elongation $\kappa=1.62$ and triangularity $\delta=0.15$. The idea was to make the simulation result depend only on the basic discharge parameters $\left(B_{\mathrm{t}}, I_{\mathrm{p}}\right)$ and the average electron density $\bar{n}_{\mathrm{e}}$, and not on particular experimental data, in order to eliminate measurement uncertainty variation and minor effects contributions. The implemented generalization of boundary conditions gives good agreement with the measurements in the whole range of investigated plasma conditions.

The model for radiated power assumes tungsten as the main source, and the total radiated power amounts to $20-40 \%$ of the Ohmic power (see fig. 11). Tungsten is considered a trace impurity and does not affect the other species' densities. 
ASTRA also computes the magnetic equilibrium and the current diffusion. The current density profile evolves freely to establish an equilibrium. A model for sawtooth instability (current density redistribution, similar to [12]) is used, in order to limit the current profile peaking and the subsequent decrease of $q$ in the core. The radial position of the $q=1$ surface is defined by the equilibrium solver. The accompanying central flattening of the density and temperatures profiles is achieved with an additional term in heat conductivities and particle diffusion, that vanishes outside $r / a \approx 0.25$. We also put a correction for $\chi_{\mathrm{e}}, \chi_{\mathrm{i}}$ and $D_{\mathrm{n}}$ outside of $r / a=0.8$, in order to account for edge effects not captured by TGLF. Both modifications do not affect the radial region between $r / a=0.25$ and 0.8 , where our modeling of the turbulence is considered valid.

The TGLF setting is as follows. We work in realistic Miller geometry, with kinetic electrons. The total number of modes is 24 , in the range $k_{y} \rho_{i}=0.1-24$ (15 of them are in the short wavelength region). The heat flux is computed from two most unstable modes. The velocity shearing effects have been tested and found small in these conditions, because the intrinsic $V_{E \times B}$ and $V_{\|}$rotation velocities (no NBI) do not produce strong velocity shear. This has been shown in both TGLF and GKW simulations. Hence, the shearing has been excluded from the modeling for simplicity. We use the saturation rule 1 [13], though both 0 and 1 have been tested, with only a little difference.

\subsection{Effective charge scaling}

Impurity concentration has been identified as an important quantity for ITG turbulence [14-16]. Increased impurity content leads to main ion dilution, which reduces ITG drive. In the experiment, $Z_{\text {eff }}$ information is available either from the Bremmstrahlung background measurements or active CXRS measurements of low$\mathrm{Z}$ impurity species. Unfortunately, the Bremmstrahlung measurements are often corrupted by reflections, and low-Z impurity measurements are not routinely available in discharges using NBI blips only to measure rotation. On the other hand, the loop voltage is routinely measured with high temporal resolution, and it depends on the effective charge via plasma conductivity. Therefore, we have made an estimation of the $Z_{\text {eff }}$ dependence on engineering parameters of the plasma that would match the experimental loop voltage. The resulting scaling has the form

$$
Z_{\mathrm{eff}}=1+3.2 I_{\mathrm{p}}^{3} / \bar{n}_{\mathrm{e}}^{3}
$$

with $I_{\mathrm{p}}$ the total plasma current in $M A$ and $\bar{n}_{\mathrm{e}}$ the line averaged electron density in $10^{19} \mathrm{~m}^{-3}$.

Fig. 2 shows the scaling $Z_{\text {eff }}$ values of the modeling compared to the $Z_{\text {eff }}$ from the loop voltage measurements data taken from the AUG Ohmic L-mode database. This scaling is used for all the simulations in this work. The scaling values can be compared to $Z_{\text {eff }}$ computed from direct boron density measurements with CXRS in a different discharge with similar parameters, and the ranges of values are similar. This supports the estimation that boron is the main impurity species, while others only present in small amounts. 

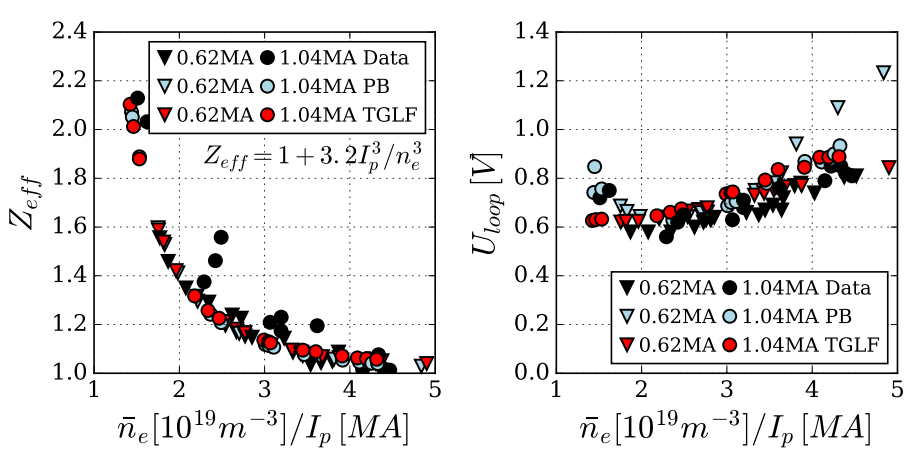

Figure 2. Left: Comparison of measured $Z_{\mathrm{eff}}$ (Data) to the scaling (PB and TGLF) for the two plasma current values, as a function of $n_{\mathrm{e}} / I_{\mathrm{p}}$. Right: corresponding loop voltage values.

Note that here and in the following, the last three points of the $I_{\mathrm{p}}=0.62 \mathrm{MA}$ data subset belong to the second discharge in the subset, \#28243, which had slightly different conditions, than the first one, \#28386. Presumably, the position of the $q=1$ surface plays a role here, in the discharge \#28243 it is closer to the magnetic axis. These differences are not accounted for, hence not visible, in the ASTRA-TGLF modeling, but are often present in the power balance simulation results.

\section{Simulation results}

As already mentioned, the data subsets represent density ramps at two different plasma currents, so that in each subset plasma evolves from the LOC to the SOC regime. Figures 3 and 4 show simulated plasma kinetic profiles in comparison with the measurements for both subsets of discharges. The profiles corresponding to the LOC phase are shown in blue, the SOC - in red, and those at the transition between the phases are drawn black.

The simulated and measured electron density profiles (subfig. b) demonstrate a very good agreement, and the simulated electron temperatures $(a)$ deviate only slightly from the experimental. Simulated ion temperature profiles remain the same during the density growth, and while TGLF reproduces the measurements well at low densities, a deviation appears at high density, where the observations show a decrease of $T_{\mathrm{i}}$. This difference is significant, up to $30 \%$. It is also reflected in the simulated pressure profiles, which are higher then experimental in the SOC regime. The effect this deviation may produce is discussed furter in this section.

In order to look in more detail at the evolution of the kinetic profiles as the density increases, we show a comparison of measured and simulated values in the core, $r / a=0.2$ and at the edge, $r / a=0.8$, for both subsets of data, see Fig. 5 .

The interesting part here is the evolution of the plasma pressure, which is also the plasma kinetic energy. In the experiment, the edge pressure grows steadily together with the electron density, while in the core it saturates at a certain point. In the plasmas with $I_{\mathrm{p}}=0.62 \mathrm{MA}$ the saturation point is at around $2.0 \times 10^{19} \mathrm{~m}^{-3}$, for the plasma 

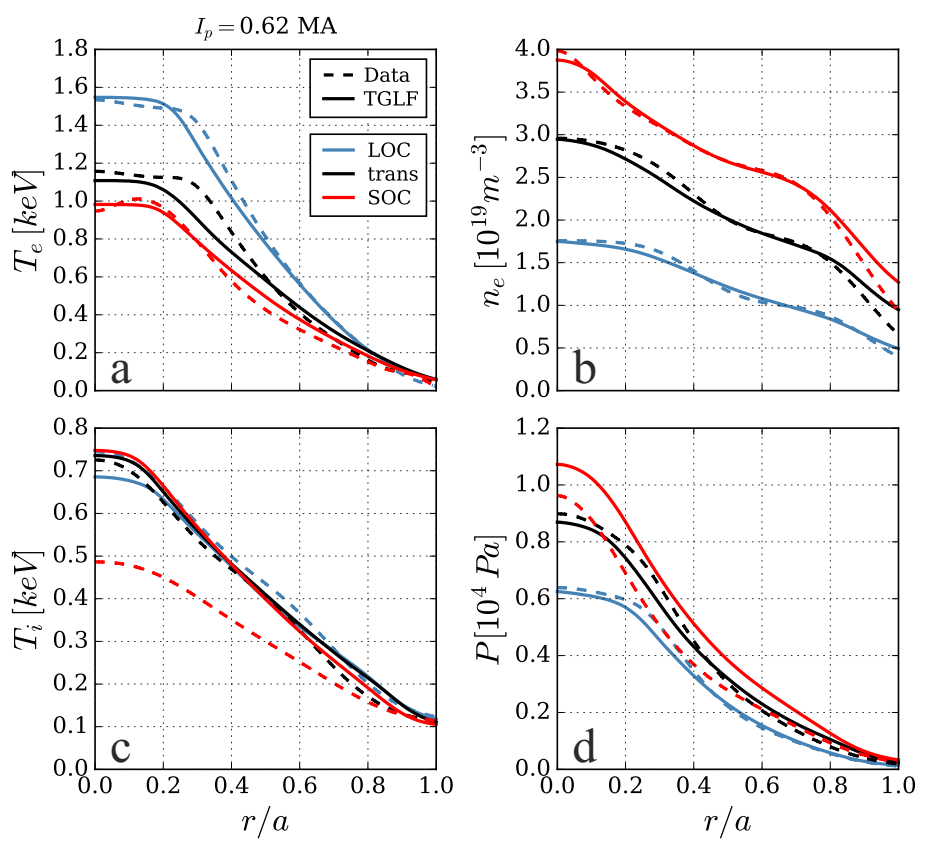

Figure 3. Kinetic plasma profiles simulated with ASTRA-TGLF (solid) and the corresponding experimental measurements (dashed) in the LOC regime, at the transition critical density, and in the $S O C$ regime for $I_{\mathrm{p}}=0.62 \mathrm{MA}$.
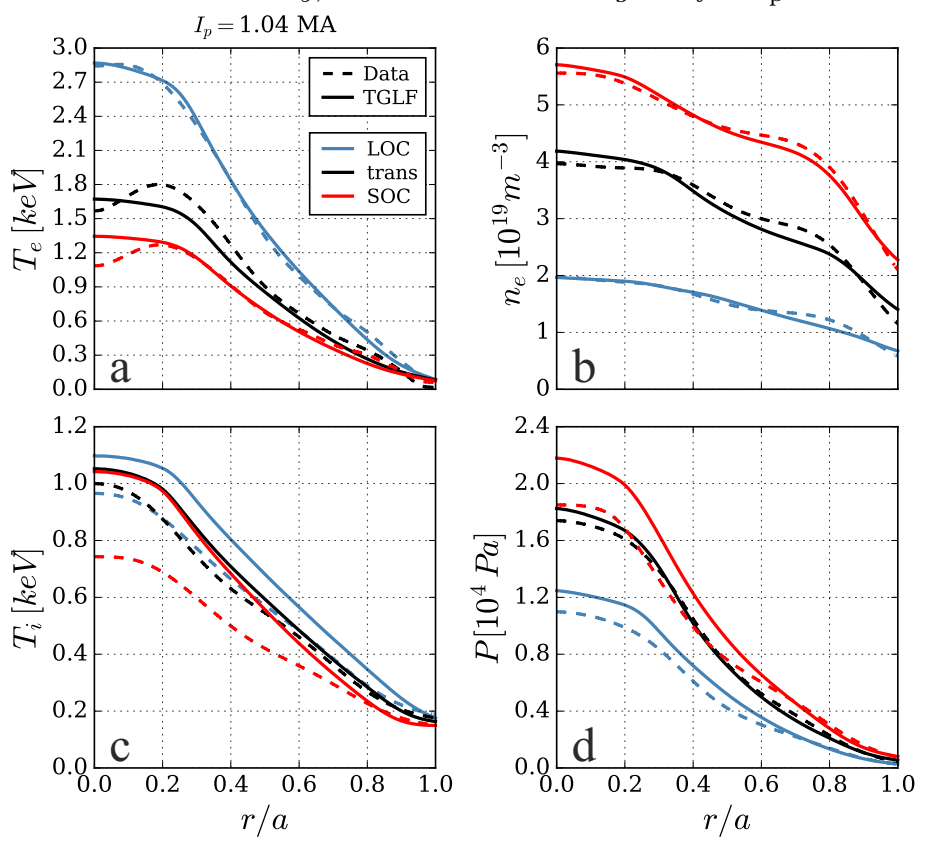

Figure 4. Same as above for $I_{\mathrm{p}}=1.04 M A$.

with $I_{\mathrm{p}}=1.04 \mathrm{MA}$ it is roughly $3.1 \times 10^{19} \mathrm{~m}^{-3}$. These points can be associated with the LOC-SOC transition critical density. It can be seen that, while the core electron temperature reduces gradually as the density increases, the core ion temperature stays rather constant before the critical density and starts to decrease right after it is reached. Therefore, the plasma energy saturation is likely caused by the ion temperature behavior.

In the simulation, the pressure saturation at the transition density is less 

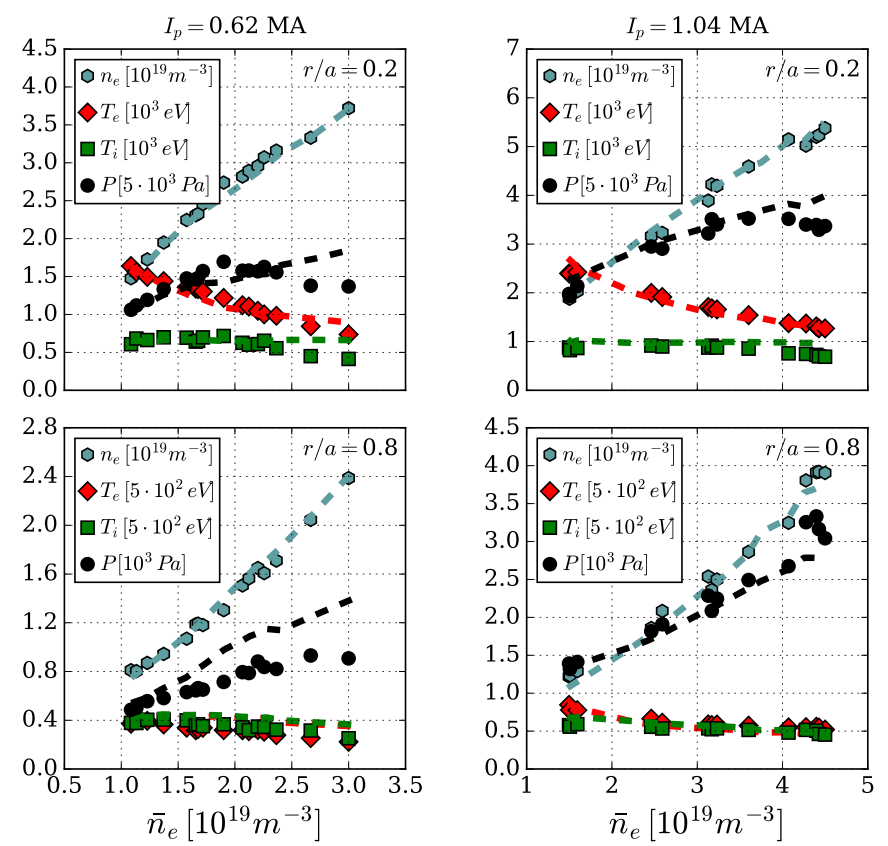

Figure 5. Evolution of plasma kinetic parameters in the core $(r / a=0.2$, top row) and at the edge $(r / a=0.8$, bottom row) with the density growth, comparison between $P B$ (points) and TGLF (lines) simulations with ASTRA.
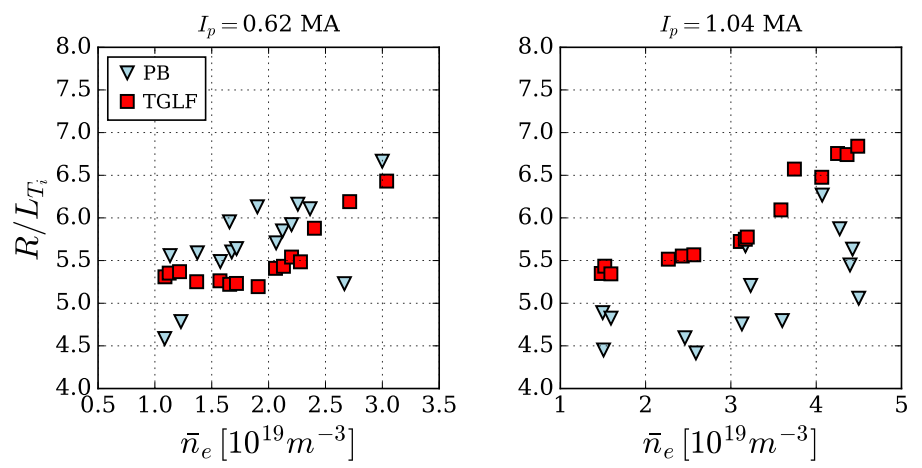

Figure 6. Normalized logarithmic gradient of ion temperature $R / L_{T_{\mathrm{i}}}$, averaged between $r / a=0.4$ and 0.6 , as a function of mean electron density.

pronounced, because of almost no reduction in the core ion temperature, while the electron temperature and density are reproduced well. And although there is a discrepancy in the ion temperature, Fig. 6 shows that the normalized logarithmic gradient of ion temperature is actually close to the experimental values.

Fig. 7 compares the experimental and the simulated temperature ratio $T_{i} / T_{e}$, averaged around mid-radius, which due to the similarity of $T_{e}$ and $T_{i}$ profiles is a good proxi for the global temperature ratio. There is a deviation at $I_{\mathrm{p}}=1.04 M \mathrm{~A}$, high density, where the simuated value is increasing towards 0.8 , while in the experiment it stays at 0.7 .

The effect of $T_{i} / T_{e}$ on turbulence frequency at this high density at a given radial position $r / a=0.55$ is shown in Fig. 8. Certainly, the difference between 0.7 and 0.8 is rather small. Therefore, we consider the overall effect of the ion temperature mismatch between the modeling and the measurements to be negligible. 

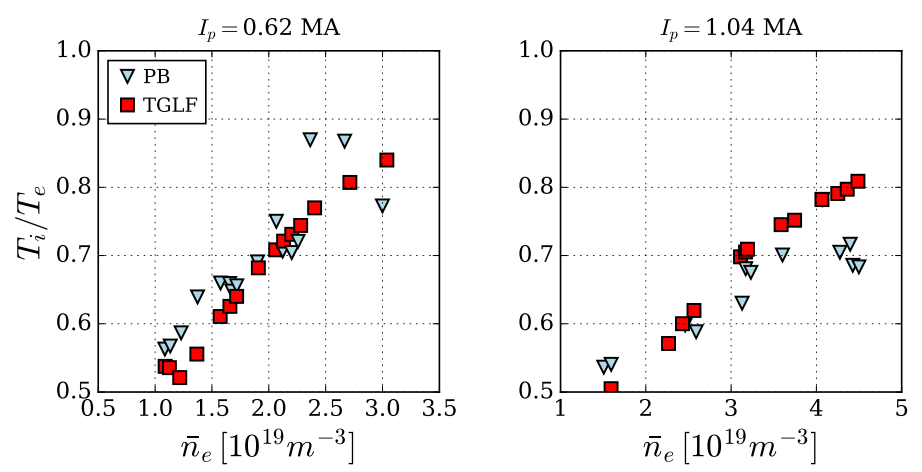

Figure 7. Temperature ratio $T_{i} / T_{e}$, averaged between $r / a=0.4$ and 0.6 , as a function of mean electron density.

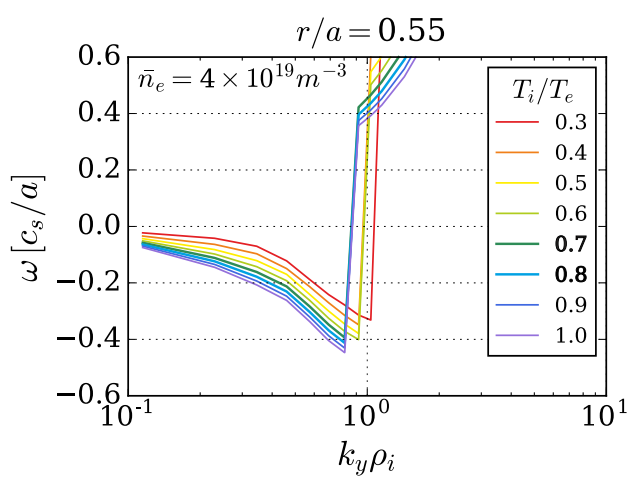

Figure 8. Temperature ratio $T_{i} / T_{e}$ effect on turbulence frequency at fixed electron density.

\subsection{Heat conductivities analysis}

We continue the analysis by comparing the electron and ion heat conductivities, $\chi_{\mathrm{e}}$ and $\chi_{\mathrm{i}}$, from PB and TGLF simulations. In the power balance simulations the heat conductivities are computed from the experimental temperature profiles. In the selfconsistent ones, the total heat conductivity is a sum of the turbulent, computed by TGLF, and neoclassical contributions. In all regimes, the neoclassical contribution is approximately one order of magnitude smaller then the turbulent. Fig. 9 illustrates the comparison of heat conductivities, presenting the values averaged over the radial range from $r / a=0.4$ to 0.6 .

The TGLF electron heat conductivity shows inverse proportinality to the average electron density, reducing from slightly above $1 \mathrm{~m}^{2} / \mathrm{s}$ at $1 \times 10^{19} \mathrm{~m}^{-3}$ to $0.4 \mathrm{~m}^{2} / \mathrm{s}$ at high density for both plasma currents. The power balance values show a similar trend at low and middle density values, while at high density they significantly diverge. The ion heat conductivity from TGLF demonstrates a slight decrease with electron density increase as well, except for the very low densities, where it grows. On the contrary, the $\chi_{\mathrm{i}}$ from power balance shows a steady increase, roughly quandratic with the average density, reaching up to $4 \mathrm{~m}^{2} / \mathrm{s}$ at the highest of our density values in both cases.

The values of power balance $\chi_{\mathrm{e}}$ and $\chi_{\mathrm{i}}$ depend on the assumptions made for the model of radiated power and the magnetic equilibrium. In extreme cases, a variation up 

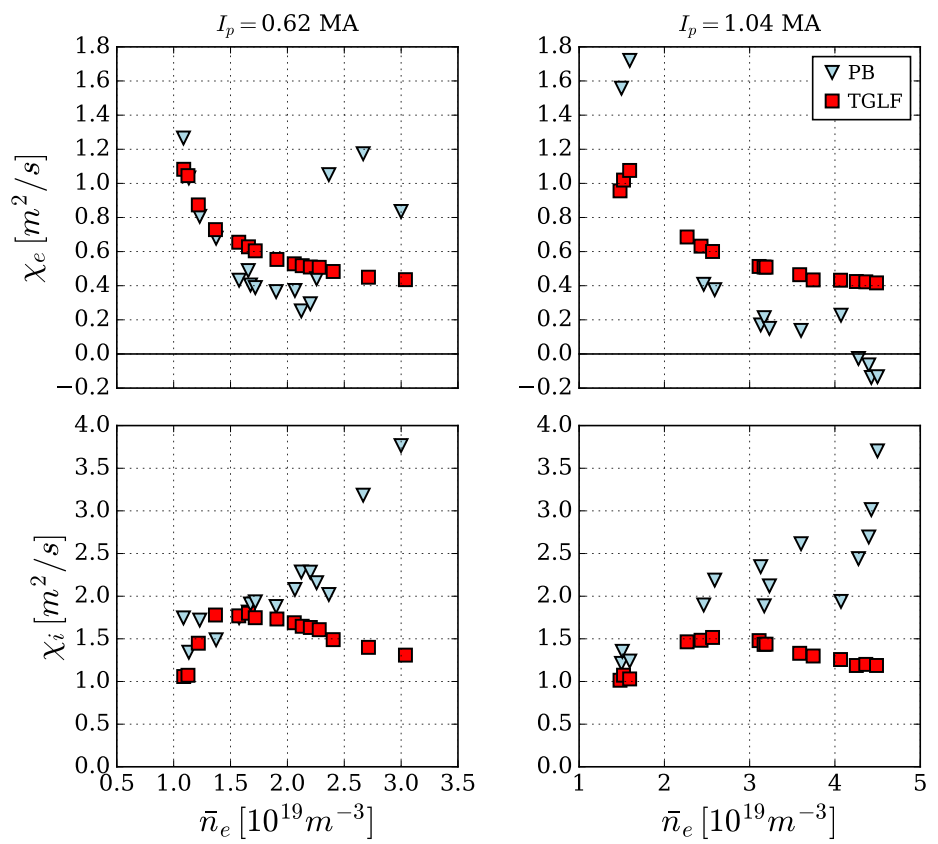

Figure 9. Heat conductivities from $P B$ and TGLF simulations, averaged between $r / a=0.4$ and 0.6 as functions of mean electron density.

to $30 \%$ is observed in both directions, and the $\chi_{\mathrm{e}}$ trend may vary. However, the rapid rise of $\chi_{\mathrm{i}}$ at high density retains.

\subsection{Energy confinement time}
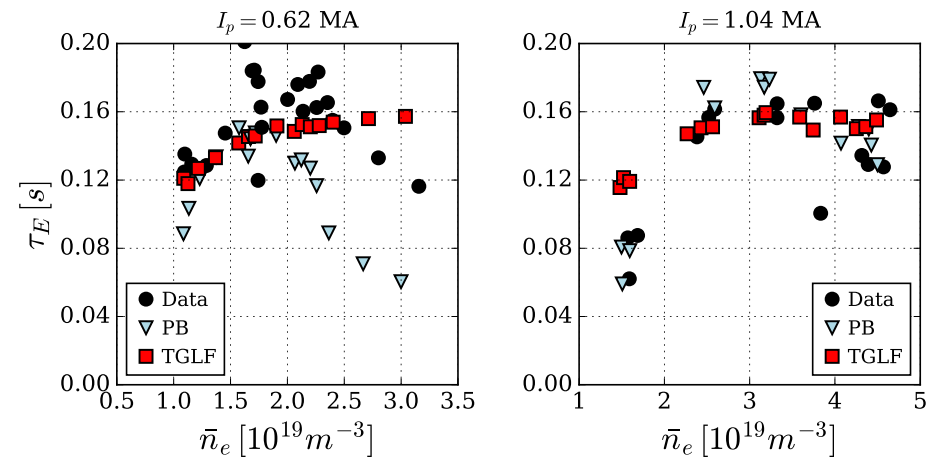

Figure 10. Energy confinement time as a function of average electron density.

The global energy confinement time for all considered discharges is displayed in Fig. 10, where the experimental estimate is compared to the values from PB and TGLF simulations. Despite the similarity of the kinetic plasma profiles in the experimental database and the PB modeling, the energy confinement time values do not always coincide. The reason is, first of all, in the different approaches to compute $\tau_{\mathrm{E}}$. The experimental estimate takes the MHD energy $W_{\mathrm{MHD}}$ as the stored energy, while in ASTRA it is the total kinetic energy. Also, although the Ohmic power is defined as 
$P_{\mathrm{Ohm}}=I_{\mathrm{p}} U_{\text {loop }}$ in both approaches, the loop voltage values slightly differ, as discussed in section 2.2 .

Looking to the TGLF simulations results, one can see that the order of magnitude is recovered by the model. This implies that, as the kinetic profiles are satisfactorily reproduced (see figures 3,4 ), also the net power $P_{\mathrm{Ohm}}-P_{\mathrm{rad}}$ is obtained within reasonable limits, despite uncertainties in the effective charge, loop voltage, and radiated power. Since the former two could be reasonably constrained by measurements, the radiated power approximation seems to be good, as well.

Second, the trend of $\tau_{\mathrm{E}}$ with respect to the electron line averaged density shows both the linear and the saturated phase. Note that the saturated phase begins at lower densities $\left(\approx 2 \times 10^{19}\right)$ for the lower current case, while the 1 MA case displays the transition at $\approx 3.1 \times 10^{19}$, and here the simulations and the measurements agree.

While in the higher current case the experimental confinement time in the SOC phase experiences minor to no degradation, in the lower curren case it is significantly reduced after the phase transition. The simulations results do not have this feature, they show confinement time saturation at a constant level.

\subsection{Detailed analysis of global energy and power balance}
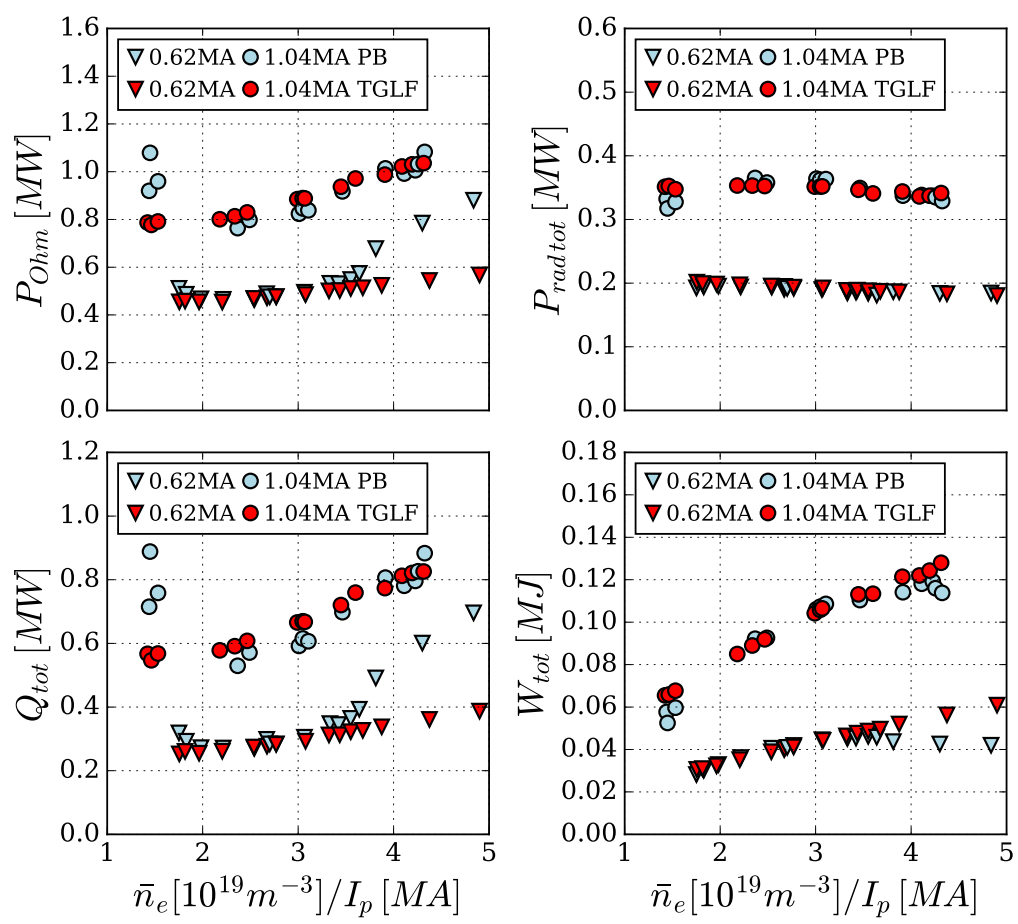

Figure 11. The Ohmic power $P_{\text {Ohm }}$, total radiated power $P_{\text {rad tot }}$, total absorbed power $Q_{\text {tot }}$ and total plasma energy $W_{\text {tot }}$ as functions of the ratio $\bar{n}_{\mathrm{e}} / I_{\mathrm{p}}$.

Since the confinement time is the ratio between plasma energy and absorbed power, it is useful to look at these ingredients separately and compare the modeling results to 
the experimental data. In Fig. 11, the ASTRA-TGLF simulated Ohmic power $P_{\mathrm{Ohm}}$, total radiated power $P_{\text {rad tot }}$, total absorbed power $Q_{\text {tot }}$ and total plasma energy $W_{\text {tot }}$ as functions of the ratio $\bar{n}_{\mathrm{e}} / I_{\mathrm{p}}$ are compared to the results with $\mathrm{PB}$.

Considering the accuracy of loop voltage agreement (Fig. 2), the Ohmic power is reproduced with the same quality. So is $Q_{\text {tot }}$, which is an important element to compute the power balance. It is shown to moderately increase with the density. The plasma energy grows roughly linearly with the density, with simulated values higher than experimental at high densities, which is a consequence of underestimated ion transport, as shown in Fig. 9.

Taking into account the discrepancies in experimental and simulated $W$ and $Q$, the ratio of $W / Q$ explains the behavior of the confinement time shown in Fig. 10, including the big deviation seen in the SOC phase for the lower current case.

\subsection{Density peaking}
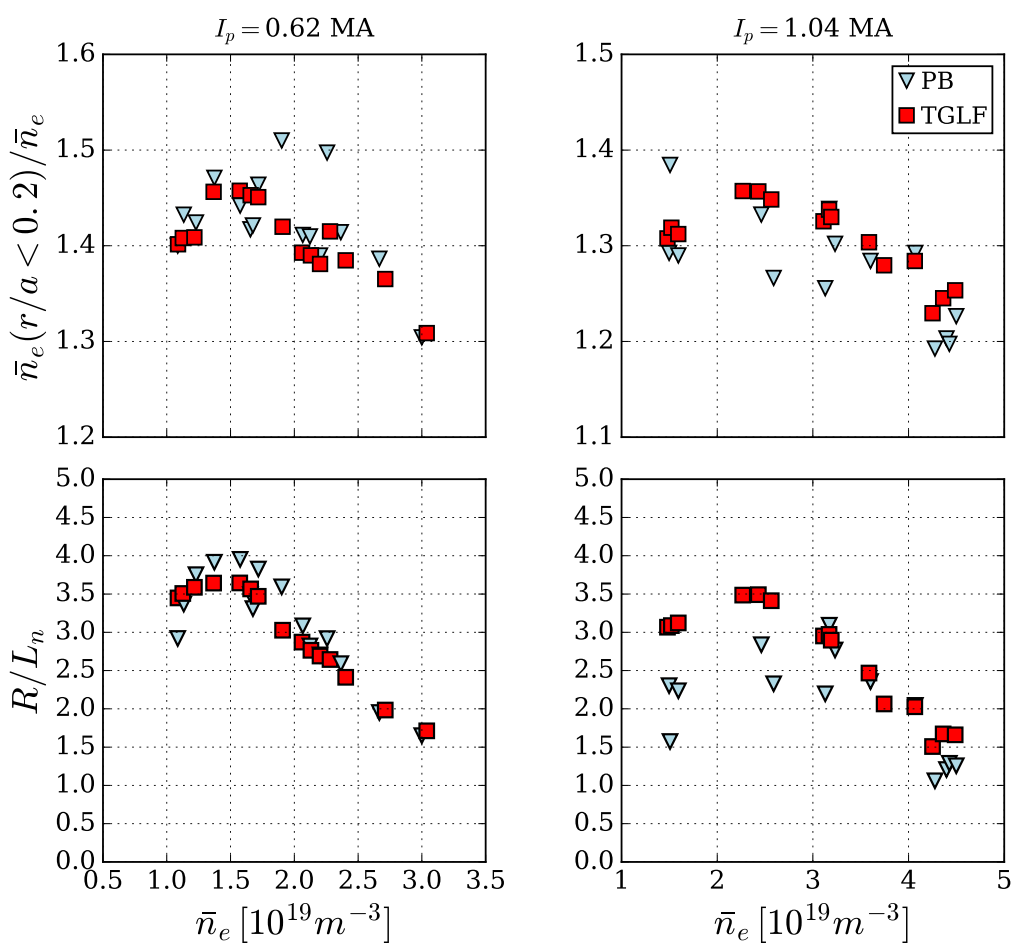

Figure 12. Measures of density peaking: the ratio $n_{e}($ core $) / \bar{n}_{e}$ (top) and the normalized logarithmic electron density gradient averaged between $r / a=0.3$ and $r / a=0.7$ (bottom) as functions of average electron density, for the two plasma currents.

L-mode Ohmic plasmas also demonstrate a change in density profile peaking behavior at different average electron densities. The profile is rather flat at very low density, it becomes peaked shortly before the critical density of the LOC-SOC transition, and shifts toward a flat-top shape as the density is further increased. The peaking has been attributed to TEM dominance, while the profile flattening occurs as ITG becomes 
dominant $[4,18,19]$. Fig. 12 shows the evolution of density peaking during density rampups in our plasma conditions, as defined by the ratio of the centrally averaged electron density (inside $r / a=0.2$ ) to the full volume average value, and by a $R / L_{n}$ averaged around the mid-radius, between $r / a=0.3$ and $r / a=0.7$.

Note that the highest peaking appears at the average densities below the LOC-SOC transition levels. This is consistent with the result that the dominant turbulence regime is already the ITG when the LOC SOC transition takes place, whereas dominant TEM conditions can occur at densities which are below those corresponding to the maximum density peaking. However, the situation can be different in other machines, as reported in $[20]$.

\section{Dedicated analysis of turbulence spectra}

In order to gain more understanding of how the energy transport varies during the density buildup, it is instructive to look at the spectral characteristics of the turbulence as predicted by TGLF. For this analysis, the TGLF code is run separately, outside of ASTRA environment with data files generated in ASTRA-TGLF modeling for specific radial points. It produces $k$-spectra of the most unstable mode real frequency $\omega(k)$ normalized to $c_{\mathrm{s}} / a$, and the electron and ion heat fluxes $Q_{\mathrm{e}, \mathrm{i}}(k)$ normalized to gyroBohm units. Note that TGLF could be run directly with experimental data, though the ASTRA-TGLF outcome is preferable, since all plasma parameters are smooth and exactly consistent in that data, and the values are still very close to experimental. One could argue that the simulated ion temperature much exceeds the measurements, but what matters is the normalized gradient $R / L_{T_{\mathrm{i}}}$, and Fig. 6 demonstrates that it is reproduced relatively well. In this situation, the only turbulence-relevant parameter outside the experimental error bars is the temperature ratio $T_{\mathrm{i}} / T_{\mathrm{e}}$. The effect this and other quantities have on transport is discussed in section 4.1.

Fig. 13 illustrates the turbulence spectral analysis for the data subset with $I_{\mathrm{p}}=$ $1.04 M A$, presenting $\omega, Q_{\mathrm{e}}$ and $Q_{\mathrm{i}}$ in the range $k_{\mathrm{y}} \rho_{\mathrm{s}}=[0.1-2]$. Electron heat transport due to high-k turbulence has been found small according to TGLF in these plasma conditions, it does not exceed $5 \%$ of the total electron transport. Therefore we limit the $\mathrm{k}$-space shown in the plots to $k_{y} \rho_{s}<2$. We choose the radial points $r / a=0.5$ and $r / a=0.7$, so that we are able to show also the radial variation of the parameters presented. The picture for the lower current case is very similar, scaled to the averaged density by the plasma current ratio. For the mode frequency $\omega$, the sign convention is that positive value refers to a mode rotating in the electron diamagnetic direction (e.g., TEM when the frequency is robustly positive).

It can be seen from the top plots $(a, b)$, that the transition between TEM and ITG indeed happens along the density scan (line averaged density as a control parameter in the horizontal axis). However, the scales below $k_{\mathrm{y}} \rho_{\mathrm{s}}=0.2$ seem to be dominated by ITG already at the lowest densities in the range of study, and other wavenumbers up to $k_{\mathrm{y}} \rho_{\mathrm{s}}=1.0$ experience the mode transition at different values of $\bar{n}_{\mathrm{e}}$. As a rule, 
lower $k_{y}$ transit first, since the collisional frequency is wavenumber independent, so at a fixed collisionality, electron detrapping at larger scales is more efficient [21]. Comparing the two radial positions in the range $k_{\mathrm{y}} \rho_{\mathrm{s}}=[0.6-1]$, one sees that outer regions of plasma switch the dominant mode to ITG at lower densities, due to higher collisionality and $R / L_{T_{\mathrm{i}}}$ at the edge. Interestingly, the mode transition at scales responsible for the dominant heat transport (namely, $k_{\mathrm{y}} \rho_{\mathrm{s}}=[0.3-0.7]$ ) occurs close to the average density of the highest density peaking, especially at the mid-radius. At the same time, no significant variation of the $\omega$ spectrum is observed at the LOC to SOC regime transition. It should be noted here, that the boundary between positive and negative fequencies is not exact and completely stable, it may fluctuate where the fequency absolute value is close to zero.

The electron heat flux $Q_{\mathrm{e}}(k)$ in the subplots $c$ and $d$ is expressed in real units $\left([G B] \cdot n T^{5 / 2}\right)$, in logarithmic scale, in order to resolve also minor contributions of subdominant modes. The white line marks the boundary between positive and negative
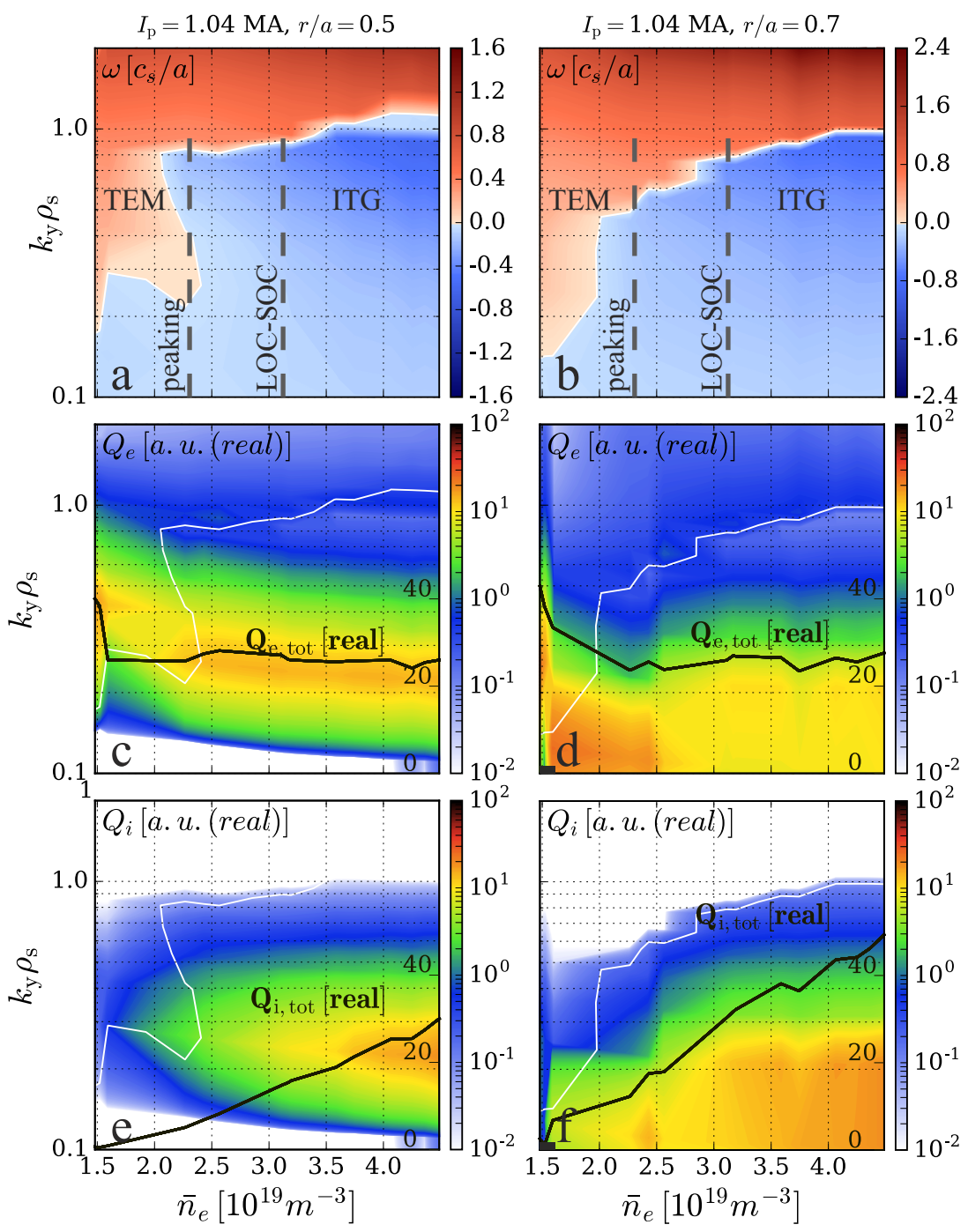

Figure 13. Turbulence spectral characteristics. 
mode frequencies. Clearly, the electron heat flux at a given scale drops dramatically as this scale shifts to ITG-dominant regime, since heat transport becomes non-resonant. Nevertheless, the electron heat transport channel in the ITG domain is still substantial, and the total heat flux (shown with the black line) stays relatively constant. Interesting to note, that the wavenumber of the mode with highest transport activity shifts towards larger scales as the average density increases, as well as when comparing outer regions of plasma to mid-radius position.

The ion heat flux (subplots $e$ and $f$ ) is related to ITG alone, and no transport is observed outside the typical scales of ion turbulence. The plot $e$ shows that ITG activity starts already at the lowest density in the study range, but the ITG mode is still subdominant there. The total heat flux in the ion channel increases steadily with the average density, much due to increasing strength of the ITG mode with decreasing $Z_{\text {eff }}$.

This analysis confirms the relation of the TEM-ITG dominant turbulent mode transition to the density peaking phenomenon. At the same time, we find no proof that this mode transition impacts the global confinement and, more specifically, leads to the energy confinement time saturation. Rather, it seems that it is the ITG-driven ion heat flux that reaches certain value, above which the confinement does not improve anymore. Similar conclusions have been made in $[6,10]$, but these results contradict $[22,23]$.

\subsection{Sensitivity analysis of heat fluxes}

In order to complete the study of heat transport in electron and ion channels, we perform a sensitivity analysis of these quantities to various plasma parameters. Since the average electron density and the plasma current (that influences also the magnetic equilibrium) are of no doubt important quantities, we fix one of them by the choice of data points at play.

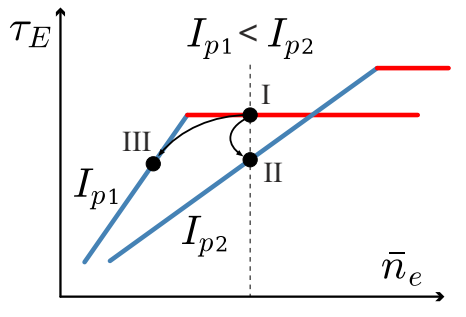

Figure 14.

Substitution scheme First, we define a data point (I) in the subset with $I_{\mathrm{p}}=$ $0.62 M A$ in the beginning of the SOC phase, so that in the subset with $I_{\mathrm{p}}=1.04 M A$ there exist a data point (II) with the same average density, but in LOC phase. We then find a third point (III) in the lower current subset, so that its confinement time equals to that of the point II (see Fig. 14). During the self-consistent ASTRA-TGLF simulations for each of these three data points a TGLF input file is produced, that corresponds to a specific radial location (here $r / a=0.5$ ). These input files contain all the physics parameters needed to run the code, like safety factor, magnetic shear, normalized temperature and density gradients, species density concentrations etc. We than take the data file for the point II and replace one of the parameters with that from the point I, keeping all the others unchanged. As a result, we simulate plasma conditions of the LOC regime, with one of the parameters from the SOC. Running the TGLF code with this modified input file and comparing the 
outcome in terms of heat fluxes, one can see which parameters are mostly responsible for the transition between the LOC and SOC plasma turbulence regimes. The same is done with the LOC data of the point III. This way we arrive to two sets of modified plasma conditions and the heat fluxes obtained accordingly.
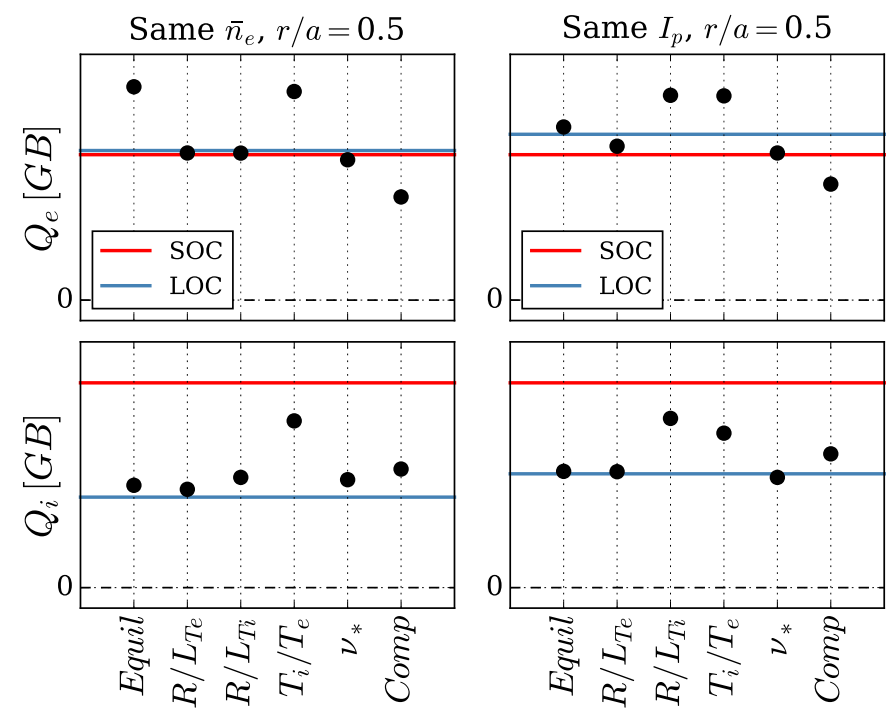

Figure 15. Sensitivity of electron and ion conductivities to single parameter variation.

Fig. 15 presents the result of this exercise, showing relative variations of the electron and ion heat fluxes in gyro-Bohm units. The lines mark the heat fluxes of pure plasma conditions: red corresponds to the SOC regime of I, blue on the left ("Same $\bar{n}_{\mathrm{e}}$ ") is the LOC of II, blue on the right ("Same $I_{\mathrm{p}}$ ") represents the LOC of the data point III. Note that the electron heat flux is almost at the same level in all three conditions. The replaced parameters are: magnetic equilibrium and plasma geometry Equil, normalized logarithmic gradients of electron and ion temperatures, the temperature ratio, collisionality, and the plasma composition Comp, that includes species densities and density gradients. The black dots show the heat fluxes levels in the conditions when this parameter is replaced. A dot that lies on the blue line means that this replacement has no effect. An important remark: if one replaces all of these parameters at once, both heat fluxes appear at the red lines.

The results can be derived as follows:

(i) Having different currents or different densities does not have a direct impact on the transport levels, as the plots look very similar, except for the effect of changed equilibrium on the electron heat flux when the averaged density is fixed, which is however expected.

(ii) Reducing the electron-ion temperature difference raises $Q_{\mathrm{e}}$ due to TEM destabilisation, whereas increasing the collisionality has the opposite effect. $R / L_{T_{\mathrm{i}}}$ affects ITG-driven electron heat transport. The Comp factor impacts $Q_{\mathrm{e}}$ via the electron density gradient, that is smaller in SOC. 
(iii) $Q_{\mathrm{i}}$ is influenced the most by $R / L_{T_{\mathrm{i}}}$ and $T_{\mathrm{i}} / T_{\mathrm{e}}$ factors that have effect on ITG stability, and by the impurity concentration $\left(Z_{\text {eff }}\right)$ embedded in the Comp factor. High impurity content means dilution of main ion species that reduces ITG drive at lower average electron densities.

We can conclude here that the $Z_{\text {eff }}$ impact on ITG turbulence has proven an important factor for the ion heat flux variation over plasma density scan, which leads to LOC-SOC regime transition, while the electron heat flux has no preferential change. The role of $Z_{\text {eff }}$ in determining a correct behavior of the ITG along the LOC-SOC transition has also been previously discussed [14-16].

\section{Effect of impurity content on turbulence and confinement}

In order to show the effect of the impurity concentration on turbulence more explicitly, and inspired by numerous experiments on achieving so-called improved Ohmic confinement (IOC) phase in Ohmic plasmas (for example, as described in [24,25]), we have also conducted ASTRA-TGLF simulations with various $Z_{\text {eff }}$. We use the same modeling approach as discussed in section 2.1. With fixed $Z_{\text {eff }}$ dependence on $\bar{n}_{e}$ and flat $Z_{\text {eff }}$ radial profile, we investigate the range from $Z_{\text {eff }}=1$, i.e. pure deuterium plasma, to $Z_{\text {eff }}=4$ in the Ohmic plasmas with $I_{\mathrm{p}}=1.04 M A$. The results can therefore be directly compared to those presented above for the same plasma current. Note that while values with $Z_{\text {eff }} \geq 3$ are hardly realistic with boron impurities alone, they may be seen as an extension of the realistic range, which shows that the trend of energy confinement improvement has its limits.
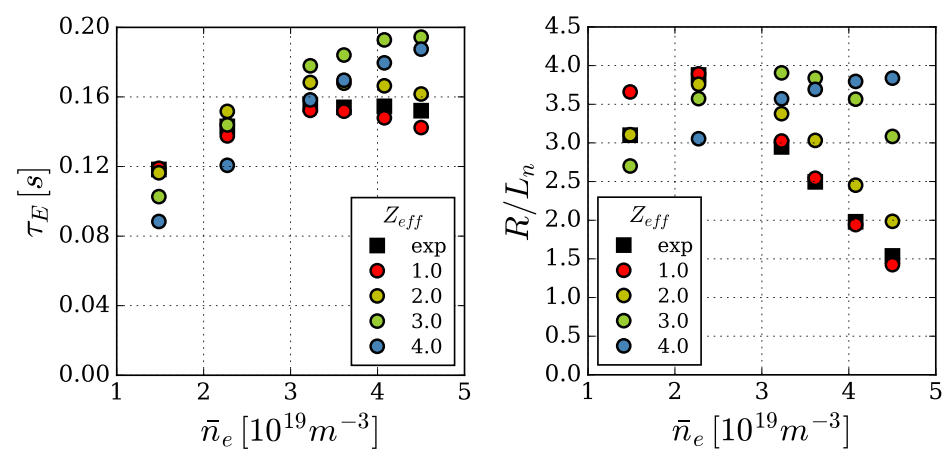

Figure 16. Energy confinement time $\tau_{\mathrm{E}}$ and the normalized logarithmic electron density gradient $R / L_{n}$ averaged within the radial range $r / a=0.4-0.6$ as functions of average electron density, for different $Z_{\text {eff }}$ values.

Fig. 16 compares the energy confinement time $\tau_{\mathrm{E}}$ and the mid-radius $R / L_{n}$ as functions of the average electron density for the plasma with the experimental $Z_{\text {eff }}$ scaling discussed in pervious sections (shown with black squares), to those of plasmas with other $Z_{\text {eff }}$ values (colored circles). It can be seen from the $\tau_{\mathrm{E}}$ plot that with increasing the boron content the linear phase extends itself to higher densities, and the $\tau_{\mathrm{E}}$ saturation becomes less pronounced. The best confinement at high density is 

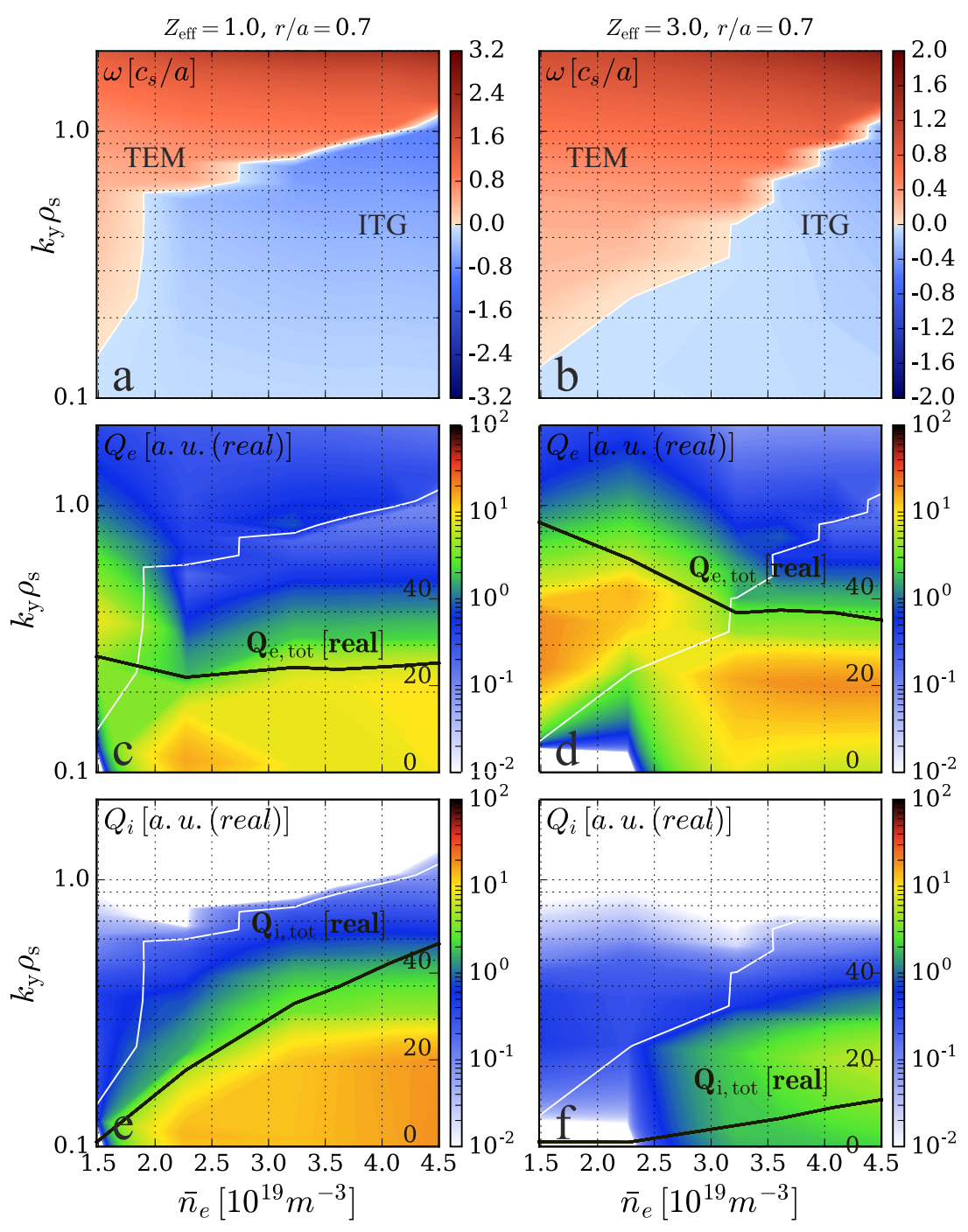

Figure 17. Turbulence spectral characteristics with $Z_{\mathrm{eff}}=1(a, c, e)$ and $Z_{\mathrm{eff}}=3$ $(b, d, f)$.

reached with $Z_{\text {eff }} \approx 3$, when no saturated phase can be distinguished anymore, while further increase of the impurity concentration reduces the confinement quality. At this high $Z_{\text {eff }}$ the negative effect of reduced ion density prevails over the positive effect of ITG turbulence suppression. The shift of the $R / L_{n}$ peak to higher density with the $Z_{\text {eff }}$ increase serves as an indicator for the corresponding shift in the dominant turbulent mode transition, as discussed in section 3.4.

Fig. 17 presents $\omega, Q_{\mathrm{e}}$ and $Q_{\mathrm{i}}$ at the radial position $r / a=0.7$ in the range $k_{\mathrm{y}} \rho_{\mathrm{s}}=[0.1-2]$, obtained with pure deuterium plasma, $Z_{\mathrm{eff}}=1.0$ (on the left), and with significant impurity content, $Z_{\text {eff }}=3$ (on the right) that corresponds to the best $\tau_{\mathrm{E}}$ as demonstrated above, in Fig. 16. Figure 17 can be compared with the Fig. 13 (b,d,f) that represents the same spectral characteristics at the same radial location for plasmas with "natural" $Z_{\text {eff }}$ scaling and can be considered as a reference. In pure deuterium plasma the ITG takes over at already very low density, that eventually leads to slightly lower $\tau_{\mathrm{E}}$ 
than in the reference case. What is more important, with $Z_{\text {eff }}=3$ the TEM dominates the heat transport up to significantly higher densities, since ion dilution effect stabilizes the ITG. Moreover, since the total ion density is low, the temperature ratio $T_{e} / T_{i}$ stays high, therefore stabilizing both TEM and ITG, that leads to lower heat fluxes in both electron and ion channels. TEM is further damped with increasing collisionality, and, as a combined effect, $Q_{\mathrm{e}}$ reduces up to $\bar{n}_{\mathrm{e}} \approx 3.2 \times 10^{19} \mathrm{~m}^{-3}$. As a result, the total turbulent heat flux stays rather constant with the density increase, and the energy confinement time demonstrates the linear trend even at high densities.

\section{Conclusions}

In this work it has been shown that TGLF, embedded in ASTRA transport solver, can reproduce the relevant features of the LOC-SOC transitions as observed in AUG. Essential ingredient is confirmed to be the impurity content, which acts through dilution to stabilize the ITG at lower densities, when impurities are more abundant. The observed scaling of the confinement time transition density as a function of plasma current thus reflects the dependence of $Z_{\text {eff }}$ on plasma density and current itself.

The LOC phase is explained with simple reduction of electron transport as the coupling between ion and electron temperature becomes stronger, while the SOC phase is the result of strongly stiff ion transport, which then, at high densities, does not allow the electron temperature to decouple.

The role of the transition between TEM and ITG is not directly influencing the LOC-SOC transition, however it is an essential ingredient to explain the behavior of the density profile.

We have also shown the mechanism that leads to IOC phase, when elevated impurity concentration reduces turbulent heat flux via ITG and TEM stabilization.

Acknowledgments The authors are grateful to V. Igochine and R. Fischer for discussions on sawtooth instability models, T. Odstrcil for sharing his knowledge on radiation profiles in AUG, A. Medvedeva for expert opinion on electron density profiles in AUG.

\section{References}

[1] Greenwald M et al., Phys. Rev. Lett. 53, 352 (1984)

[2] J. Rice et al., Phys. Plasmas 19, 056106 (2012)

[3] F. Romanelli et al, Nucl. Fusion 26, 1515 (1986)

[4] C. Angioni et al., Phys. Plasmas 12, 040701 (2005)

[5] C. Sung et al., Nucl. Fusion 53, 083010 (2013)

[6] C. Sung et al., Phys. Plasmas 23, 042303 (2016)

[7] G.V. Pereverzev and Y. P. Yushmanov, IPP Report 5/42 (1991)

[8] E. Fable et al., Plasma Phys. Control. Fusion 55, 12402 (2013)

[9] G.M. Staebler et al., Phys. Plasmas 12, 102508 (2005)

[10] R.M. McDermott et al., Nucl. Fusion 54, 043009 (2014)

[11] C. Angioni and O. Sauter, Physics of Plasmas 7, 1224 (2000) 
[12] Kadomtsev B.B., Sov. J. Plasma Phys. 1710 (1975)

[13] G.M. Staebler et al., Nucl. Fusion 57, 066046 (2017)

[14] M. Porkolab et al., Plasma Phys. Control. Fusion 54, 124029 (2012)

[15] J. Citrin et al. Progress in understanding quasi-coherent modes through gyrokinetic simulation Joined US-EU TTF Workshop (Culham, United Kingdom), 2014

[16] P. Ennever et al., Phys. Plasmas 22, 072507 (2015)

[17] J. Neuhauser et al., Plasma Phys. Control. Fusion 44, 855 (2002)

[18] C. Angioni et al., Phys. Rev. Lett. 107, 215003 (2011)

[19] E. Fable et al., Plasma Phys. Control. Fusion 52, 015007 (2010)

[20] O. Sauter et al., Phys. Plasmas 21, 055906 (2014)

[21] C. Angioni et al., Phys. Plasmas 16, 060702 (2009)

[22] H. Arnichand et al., Nucl. Fusion 54, 123017 (2014)

[23] H. Arnichand et al., Plasma Phys. Control. Fusion 58, 014037 (2016)

[24] F.X. Söldner et al., Phys. Rev. Lett. 61, 1105 (1988)

[25] M. Bessenrodt-Weberpals et al., Nucl. Fusion 31, 155 (1991) 